\title{
Factors associated with Depression among Patients with Breast Cancer at Moi Teaching and Referral Hospital, Eldoret, Kenya
}

\author{
Chelagat Saina $^{1}$, Benson Gakinya ${ }^{2}$ and Rogers Songole ${ }^{3^{*}}$
}

${ }^{1}$ MMED Psychiatry, Department of Mental Health, School of Medicine, Moi University, Eldoret, Kenya

${ }^{2}$ Associate Professor, Department of Mental Health, School of Medicine, Moi University, Eldoret, Kenya

${ }^{3}$ Senior Lecturer, Department of Mental Health, School of Medicine, Moi University, Eldoret, Kenya

*Corresponding author: Songole R, Senior Lecturer, Department of Mental Health, School of Medicine, Moi University, Eldoret, Kenya, Tel: +254 721208676; E-mail: rogerssongole@gmail.com

Received: January 29, 2021; Accepted: March 09, 2021; Published: March 17, 2021

\begin{abstract}
Background: Major Depression is the presence of sadness, empty or irritable mood, accompanied by somatic and cognitive changes. It is often unrecognized and untreated among patients with breast cancer, which causes amplification of physical symptoms, increased functional impairment, and poor treatment outcome. Factors associated with having depression among patients with breast cancer include age at diagnosis, tumor stage, surgery, and chemotherapy. There is limited data on depression among patients with breast cancer at Moi Teaching and Referral Hospital (MTRH).

Objective: To determine the factors associated with depression among patients with breast cancer at MTRH.

Methods: A cross-sectional study, in which Hamilton Depression Rating Scale (HAM-D) was used to diagnose and measure the severity of depression. A researcher-designed questionnaire was used to obtain socio-demographic and clinical information. The study was done at the breast cancer clinic, medical and surgical wards of MTRH, in which seventy-nine random consenting patients were interviewed from January to December 2017. Descriptive statistics were used for continuous data and categorical data. Chi-square and Fisher's exact tests were used to measuring for associations. Multivariate analysis by logistic regression was used to measure an independent association.

Results: A total of seventy-nine patients were interviewed, $98 \%$ of them were females. The mean age was $40 \pm 7.8$ years. The prevalence of depression among patients with breast cancer was 59.5\%. Those who were employed were 3 times more likely to have depression compared to the unemployed ( $\mathrm{AOR}=3.7,95 \% \mathrm{CI}: 1.07,17.27 ; \mathrm{p}=0.047)$. Patients on neo-adjuvant and palliative therapy were 9 times more likely to have depression (AOR=9.43, 95\% CI: $1.5,185.32 ; \mathrm{p}=0.044$ and AOR=9.5, 95\% CI: 1.62, 181.81; $p=0.039$ ) respectively than those on adjuvant therapy. Patients with late-stage breast cancer had $61 \%$ increased odds of having depression ( $\mathrm{AOR}=1.61,95 \% \mathrm{CI}: 0.63,4.17 ; \mathrm{p}=0.319)$ than patients with early-stage breast cancer.

Conclusion: Being employed, the use of chemotherapy, and the late stage of cancer were significantly associated with having depression.
\end{abstract}

Citation: Saina C, Gakinya B, Songole R. Factors associated with Depression among Patients with Breast Cancer at Moi Teaching and Referral Hospital, Eldoret, Kenya. J Anxiety Depress. 2021;4(1):133. 
Recommendation: Screening and interventions for depression should be initiated for all patients with breast cancer especially late stages of cancer, those on chemotherapy, and employed.

Keywords: Factors; Stages of breast cancer; Chemotherapy; Psychological syndrome

\section{Introduction}

Depression is the psychological syndrome that receives the most attention in individuals with cancer. Major depression is a frequent but unrecognized and untreated condition among breast cancer patients, which causes amplification of physical symptoms, increased functional impairment, and poor treatment adherence [1].

Breast cancer is reported to be a leading cancer diagnosis among women worldwide with more than 210,000 new cases and 40,000 deaths per year in the U.S.A. According to various studies carried out, it's been noted that the prevalence of depression among cancer patients ranges from 10\%-58\% [1,2]. Major depression was found to be as high as 38\% [1].

Many factors may contribute to depression including age at diagnosis, cancer stage, surgery, and chemotherapy [3,4]. The biological factors include the tumor burden, treatment morbidity, pain, and physical sensation. While the psychosocial factors impacting an individual functioning include, loss of control, increased dependency, change in the role of functioning, appearance, and identity [2].

Diagnosis of breast cancer might no longer be regarded as fatal but has been increasingly acknowledged as treatable. Nevertheless, cancer diagnosis and its treatment are recognized to be stressful times that underscore the need for clinicians to actually identify its psychological squeal such as depression in vulnerable patients.

Early detection and treatment of depression in cancer patients not only significantly improve their quality of life but also increase their survival rates.

According to a report by the Kenya Medical Research Institute (KEMRI), about $80 \%$ of cases of cancer are diagnosed at advanced stages, when truly little can be achieved in terms of curative treatment (National Cancer Control Strategy 2011-2016).

In the reports of the Eldoret cancer registry (2009), cancer of the cervix is the most common among females followed by breast and esophagus. In males, cancer of the esophagus at $16.5 \%$ is the commonest and it is followed by cancer of the skin $9.2 \%$, Non-Hodgkin Lymphoma 8.8\%, and prostate cancer 6.3\% respectively. In female cervical cancer is the most common at $17.4 \%$ followed by breast cancer at $13.6 \%$, esophagus at $9.2 \%$ and the least was colon-rectal cancer at $3.1 \%$ [5]. MTRH is currently handling a big number of patients with cancer (2000) in a year. The hospital has satellite clinics in North Rift and Western Kenya that helps with early detection of cancer and follow-up. 
www.yumedtext.com | February-2021 | ISSN: 2582-3264 | https://dx.doi.org/10.46527/2582-3264.133

Diagnosis of cancer, and subsequent treatment for the disease, can be an upsetting and harrowing experience that profoundly affects how the patient feels. Firstly, finding out that one had cancer can, in and of itself, make one depressed - for reasons we can all understand and empathize with. A second reason could be the unpleasant side-effects of cancer therapy. Chemotherapy, radiotherapy, and surgery, despite saving lives, all have effects that can alter our mental well-being. But the US researchers propose that chemicals produced by cancer itself could be involved. Cancer cells secrete a range of chemicals that affect how our bodies work, so it is not that far-fetched to think that they could affect how we think and feel. Pyter et al. [6] the team found from their study that Sickness behaviors are now thought to be caused by chemicals called cytokines, produced by the immune system during infection. As the researchers point out in their paper, raised levels of cytokines, at even moderate levels, have been linked to learning difficulties and emotional problems in humans. Pyter et al. [6] team thinks that low-level increases in cytokines over the period it takes for cancer to grow (months or weeks) could be enough to significantly change our emotional state. Cortisol hormones can weaken the activity of the immune system. It also has a negative-feedback effect on interleukin1 which is useful in combating some diseases.

Potential sources in the gap and inconsistencies of current knowledge include differences in the study methods (e.g., variable measures in screening or diagnosing depression), the variable definition of study populations i.e., survivors, sample selection, sampling time points, and the quality of reporting. A good review of this topic was conducted systematically [7] but did not focus on depression during survivorship; rather it looked at depression at no specific point of the disease.

\subsection{Problem statement}

The number of depression cases among patients with breast cancer at The MTRH has been rising in recent years. Depression is among the leading causes of disability worldwide. Untreated depression leads to personal suffering and increased mortality. Although the prevalence of depression varies considerably globally, the most common symptoms of depression are depressed mood, insomnia, and fatigue. Based on the estimates about 12.7 million cancer cases and 7.6 million cancer deaths are estimated to have occurred in 2008; of these, $56 \%$ of the cases and $64 \%$ of the deaths occurred in the economically developing world [8]. Cancer survival tends to be poorer in developing countries, most likely because of a combination of a late stage at diagnosis and limited access to timely and standard treatment.

A decade ago, in East Africa, an estimated 175,000 persons were living with cancer, and that number has dramatically increased, with cancer projected to become a leading cause of death in sub-Saharan Africa over the next few years. Unfortunately, as the threat of early death and disability from chronic diseases like cancer grows in sub-Saharan Africa, it is clear that countries like Kenya have very little in place to meet this challenge. According to the Eldoret cancer registry, cancer of the cervix $(17.4 \%)$ is the most common among females followed by breast (13.6\%) and esophagus (9.2\%). In males, cancer of the esophagus (16.5\%) is the commonest and it is followed by cancer of the skin (9.2\%), Non-Hodgkin Lymphoma (8.8\%), and prostate cancer $(6.3 \%)$ respectively [5]. Therefore, this brings in the fact that MTRH is handling many patients with cancer. Apart from the hospital set up they also have satellite clinics in North Rift and Western Kenya where they follow up and treat patients with cancer on specific days.

In Kenya, cancer ranks third as a cause of death after infectious diseases and cardiovascular diseases. It causes $7 \%$ of total national mortality every year. Although population-based data does not exist in the country, it is estimated that the annual 
www.yumedtext.com | February-2021 | ISSN: 2582-3264 | https://dx.doi.org/10.46527/2582-3264.133

incidence of cancer is about 28,000 cases, and the annual mortality to be over 22,000. Over $60 \%$ of those affected are below the age of 70 years. In Kenya, the risk of getting cancer before the age of 75 years is $14 \%$ while the risk of dying of cancer is estimated at $12 \%$.

\subsection{Study justification}

There is a high degree of variation among people with depression in terms of symptoms, course of illness, and response to treatment, indicating that depression may have several complex and interacting causes. However, recent advances in research technology are bringing scientists closer than ever before to characterizing the biology and physiology of depression in its different forms and to the possibility of identifying effective treatments for individuals based on symptom presentation. One of the most challenging problems in depression research and clinical practice is the treatment of depression among cancer patients in the advanced stage of the disease. Even among treatment responders, many do not have complete or lasting improvement, and adverse side effects are common. Thus, an important goal of the research is to advance the development of more effective treatments for depression among cancer patients. New figures and projections of the global cancer burden report the incidence of cancer has increased from 12.7 million to 14.1 million in 2012 . This will bring the number of cancer cases close to over 25 million next two decades.

The greatest impact will unquestionably be in low and middle-income countries which are ill-equipped to cope with the escalation in the number of people with cancer. As a result, prevention is central to reducing or reversing the rise in cancer burden. In the past few years, we were privileged to have a canter opened at MTRH: Chandaria cancer and chronic disease management canter. The center is mainly used by the Oncology unit, diabetic clinic, medical clinic, and psychiatry clinic. It is a multidisciplinary center. This has made access to treatment for various departments easily available. Chemotherapy is available and very soon the radiotherapy department will be functioning. Secondly, the study on the factors of depression among cancer patients was last carried out in 2009. I would like to know the current rate of depression is like now with the availability of all these services at MTRH.

\subsection{Objective of the study}

To determine the factors associated with development of depression among patients with breast cancer at MTRH.

\section{Methodology}

\subsection{Study design}

The study was a descriptive cross-sectional. All patients with a confirmed diagnosis of breast cancer and who meet the inclusion criteria were recruited for the study. Data obtained from the study were included, demographic and clinical data, and was to be addressed individually according to the study objectives for this study. Patients who were found to have depression were sent for review by a psychiatrist and further follow up. MTRH was chosen for the study because it is a national teaching and referral hospital that has an oncology clinic that covers the western Kenya region.

\subsection{Study area and background of the study area}

Recruitment of the study subjects was carried out at the breast cancer out-patient clinic at Chandaria cancer and chronic disease management center and in-patient's unit (surgical, medical wards) at MTRH. 


\subsection{Background of the study area}

MTRH is in Uasin-Gishu County; it is the second national referral hospital covering the western Kenya region with a population of about 16 million. It is a teaching and referral hospital used by Moi University. The hospital serves patients from western Kenya, north rift, and south rift regions. The hospital provides both inpatient and outpatient services. It handles all emergencies in the accident and emergency unit and has many specialties. These include medical, surgical, gynecological, psychiatric, pediatrics services, among others. The inpatient has a bed capacity of approximately 800 patients.

All types of cancer patients both pediatrics and adult (gynecological, surgical, and medical) patients are attended to at the center. The oncology clinic is run on Mondays and Wednesdays between 8.00 am to 2.00 pm but remains open for patients who come on any other day of the week. MTRH was chosen as a study site because it is a referral center and likely therefore to have sufficient patient numbers for the study. Patients are booked for the clinic by the record clerks. The number of patients seen with cancer in a week 150 and a year the number of patients is approximately 2000 patients. Patients with breast cancer seen in a year are about 100 .

\subsection{Study population}

The study populations were patients above 18 years seen at the out-patient clinic at MTRH, and those admitted to the medical ward with confirmed cancer of the breast and also the surgical ward admitted for surgical procedures. They should all meet the inclusion criteria.

\subsection{Eligibility criteria}

\subsubsection{Inclusion criteria}

- $\quad$ Be adults (aged 18 or older) with a definite breast cancer diagnosis.

- Patients in the medical/surgical wards with a definite breast cancer diagnosis.

- Patients at Chandaria cancer and chronic disease management center outpatient clinic with a definite diagnosis of breast cancer.

\subsubsection{Exclusion criteria}

Patients in coma' and patients with emotional instability.

\subsection{Sampling procedures}

For this study, we used census sampling.

\subsection{Sample size}

For this study we used census sampling, the number of patients seen with cancer is approximately 2000 per year. The number of patients with a histological diagnosis of breast cancer was 102 from January 2016-December 2016. My first participant was the first patient at seen at the outpatient clinic and that was in January of 2017 and the next was consecutive sampling and I completed in December of 2017. For the given period of the study, I interviewed 79 participants who meet the inclusion criteria for this study. 
www.yumedtext.com | February-2021 | ISSN: 2582-3264 | https://dx.doi.org/10.46527/2582-3264.133

\subsection{Clinical Procedures}

Patients admitted were recruited for the study within $72 \mathrm{hrs}$. The principal investigator recruited 2 research assistants who were trained by the researcher on how to carry out the study. Their main responsibility was to assist with the identification of the new patients, retrieving their files, and getting all the required information from the participants. Demographic data such as age, sex, and residential areas, level of education, and occupation achieved were recorded. A 17-item Hamilton Depression Rating Scale (HAM-D) was used to assess depression severity among patients. The total score is obtained by summing the score of each item, 0-4 (symptom is absent, mild, moderate, or severe) or 0-2 (absent, slight or trivial, clearly present). For the 17-item version, scores can range from 0 to 54. Since its development in 1960 by Dr. Max Hamilton of the University of Leeds, England, the scale has been widely used in clinical practice and became a standard in research trials. The Hamilton Depression Rating Scale has proven useful for determining the level of depression before, during, and after treatment [9]. The Ham-D has demonstrated reliability, validity, and efficiency in adults. A major review of 70 studies suggested that the internal, inter-rater, and retest reliability estimates are adequate for the global score [9]. The questionnaire is designed for adults and is used to rate the severity of their depression by probing mood, feelings of guilt, suicide ideation, insomnia, agitation or retardation, anxiety, weight loss, and somatic symptoms. The scale has been translated into several languages including French, German, Italian, Thai, and Turkish. In comparison to the Beck Depression Inventory, a meta-analysis suggested that after treatments, the HRSD was more 'sensitive to change' on retesting [10] and this is probably why it has been so widely used in clinical trials.

\subsection{Data collection and analysis}

Data was collected using a formatted questionnaire which will contain patient unique numbers for identification. The data collection forms were checked at the end of each day for completeness and inconsistencies were ruled out before data entry. Data obtained was maintained in the study computer under a password for protection. On completion of the study, data was transferred to a computer database for analysis. Descriptive statistics were used; for continuous data, means median and mode will be used, and categorical data frequencies and percentages were used. Data were analysed using STATA version 14. Descriptive statistics were done for continuous data and categorical data. Inferential statistics such as Chi-square and Fisher's exact tests were used to measure associations of categorical variables. Two sample t-tests were used to compare means of continuous variables. Logistic regression was used to measure the association between depression and socio-demographic variables.

\subsection{Ethical consideration}

Approval was sought from the institutional research and ethics committee to carry out the study. Permission and approval were sought from the chief executive officer MTRH. Patients were fully informed on all the procedures of the study and informed consent was sought from the entire study population before enrolment.

\section{Results}

\subsection{Sociodemographic characteristics}

A total of 79 respondents participated in the study. The mean age was $40(\mathrm{SD}=7.8)$ years. Of those who responded $98.7 \%$ were females and the majority were married $86.1 \%$. More than half of the respondents had achieved a tertiary level of education at $58.2 \% .79 .7 \%$ had some form of employment (TABLE 1.). 
www.yumedtext.com | February-2021 | ISSN: 2582-3264 | https://dx.doi.org/10.46527/2582-3264.133

TABLE 1. Socio-Demographic Characteristics.

\begin{tabular}{|c|c|c|}
\hline Variables & levels & $\mathbf{N}(\%)$ \\
& & $40+$ SD \\
\hline Age years & & $78(98.7)$ \\
\hline Sex & Female & $1(1.3)$ \\
\hline Marital status & Male & $68(86.1)$ \\
& Married & $11(13.9)$ \\
\hline Education levels & Never married & $4(5.1)$ \\
& Primary & $29(36.7)$ \\
& Secondary & $46(58.2)$ \\
\hline Employment & Tertiary & $63(79.7)$ \\
& Employed & $16(20.3)$ \\
\hline Substance use & Unemployed & $14(17.7)$ \\
& Alcohol & $4(5.1)$ \\
& Opiate & $1(1.3)$ \\
& Khat & $16(20.3)$ \\
\hline
\end{tabular}

\subsection{Factors associated with depression}

The second objective of the study was to determine the factors associated with depression among breast cancer patients. From the results s we had age, marital status, chemotherapy, surgical treatments, hormonal therapy, cancer stages, employment, and level of education as factors associated with depression. The results we got from this study showed that the late stage of cancer, employment, and use of chemotherapy was statistically significant $(0.002,0.047$, and 0.048$)$ respectively (TABLE 2.).

TABLE 2. Factors associated with Depression.

\begin{tabular}{|c|c|c|c|c|}
\hline Variables & Total $\mathrm{N}=79$ & $\begin{array}{l}\text { Depre =yes } \\
\mathrm{N}=32\end{array}$ & $\begin{array}{l}\text { Depre }=\mathrm{NO} \\
\mathrm{N}=47\end{array}$ & P-VALUE \\
\hline Age & $40 \pm 7.8$ & $39.5 \pm 6.8$ & $40.4 \pm 8.5$ & 0.599 \\
\hline Cancer_stg_2 & 11(13.9) & $0(0)$ & $11(23.4)$ & 0.002 \\
\hline Cancer_stg_3 & $27(34.2)$ & $13(40.6)$ & $14(29.8)$ & 0.319 \\
\hline Cancer_stg_4 & $41(51.9)$ & $19(59.4)$ & $22(46.8)$ & 0.272 \\
\hline Surgical treatment & $35(44.3)$ & $12(37.5)$ & $23(48.9)$ & 0.315 \\
\hline Hormonal therapy & $58(78.4)$ & $21(65.6)$ & $37(78.7)$ & 0.196 \\
\hline Gender female & $78(98.7)$ & $32(100)$ & $46(97.9)$ & 0.406 \\
\hline $\begin{array}{l}\text { Marital status } \\
\text { Married }\end{array}$ & $68(86.1)$ & $29(90.6)$ & $39(83)$ & 0.335 \\
\hline $\begin{array}{l}\text { Employment } \\
\text { Employed }\end{array}$ & $63(79.7)$ & $29(90.6)$ & $34(72.3)$ & 0.047 \\
\hline \multirow{5}{*}{$\begin{array}{l}\text { Substance use } \\
\text { Alcohol } \\
\text { Opiate } \\
\text { Tobacco } \\
\text { Khat }\end{array}$} & \multirow{5}{*}{$\begin{array}{l}16(20.3) \\
14(17.7) \\
4(5.1) \\
6(7.6) \\
1(1.3)\end{array}$} & \multirow{5}{*}{$\begin{array}{l}4(12.5) \\
4(12.5) \\
0(0) \\
2(6.2) \\
0(0)\end{array}$} & $12(25.5)$ & 0.167 \\
\hline & & & $10(21.3)$ & 0.38 \\
\hline & & & $4(8.5)$ & 0.143 \\
\hline & & & $4(8.5)$ & 1 \\
\hline & & & $1(2.1)$ & 1 \\
\hline \multicolumn{4}{|l|}{ Educational levels } & \multirow[b]{2}{*}{0.42} \\
\hline Primary & $4(5.1)$ & $2(6.2)$ & $2(4.3)$ & \\
\hline
\end{tabular}




\begin{tabular}{|c|c|c|c|c|}
\hline Secondary & $29(36.7)$ & $9(28.1)$ & $20(42.6)$ & \\
\hline Tertiary & $46(58.2)$ & $21(65.6)$ & $25(53.2)$ & \\
\hline \multicolumn{4}{|l|}{ Chemotherapy } & \\
\hline \multirow[b]{2}{*}{ Adjuvant chemotherapy } & $12(15.2)$ & $1(3.1)$ & $11(23.4)$ & 0.048 \\
\hline & & & & \\
\hline \multirow[b]{2}{*}{ Neoadjuvant chemotherapy } & $26(32.9)$ & $12(37.5)$ & $14(29.8)$ & \\
\hline & & & & \\
\hline Palliative Chemotherapy & 41(51.9) & $19(59.4)$ & $22(46.8)$ & \\
\hline
\end{tabular}

TABLE 3. Results of the unadjusted and adjusted analysis of depression among patients with breast cancer.

\begin{tabular}{|c|c|c|}
\hline Variable & Unadjusted ORs (95\% CIs) & P-Value \\
\hline Age & $0.98(0.93,1.04)$ & 0.609 \\
\hline Marital Status: Married & $1.98(0.52,9.65)$ & 0.342 \\
\hline Employment Status: Employed & $3.7(1.07,17.27)$ & 0.058 \\
\hline Substance Use & $0.4(0.1,1.31)$ & 0.152 \\
\hline Alcohol & $0.53(0.13,1.76)$ & 0.321 \\
\hline Tobacco & $0.72(0.09,3.92)$ & 0.711 \\
\hline Cancer Stage 3 & $1.61(0.63,4.17)$ & 0.32 \\
\hline Cancer Stage 4 & $1.66(0.67,4.19)$ & 0.274 \\
\hline Surg_Tx & $0.63(0.25,1.55)$ & 0.316 \\
\hline Hormonal Therapy & $0.52(0.18,1.42)$ & 0.199 \\
\hline Chemotherapy & & \\
\hline Adjuvant Chemotherapy & Ref & \\
\hline Neoadjuvant Chemotherapy & $9.43(1.5,185.32)$ & 0.044 \\
\hline Palliative Chemotherapy & $9.5(1.62,181.81)$ & 0.039 \\
\hline Education Level & & \\
\hline Primary & Ref & \\
\hline Secondary & $0.45(0.05,4.23)$ & 0.459 \\
\hline Tertiary & $0.84(0.09,7.49)$ & 0.867 \\
\hline
\end{tabular}

\subsection{Findings of the unadjusted odds ratio}

The findings show that there were $98 \%$ increased odds of a diagnosis of depression among patients with breast cancer among married participants, compared to the unmarried, OR: 1.98 (95\% CI: 0.52,9.65), and a 60\% reduced odds of depression among patients with breast cancer diagnosis among participants who used substances compared with those who didn't, OR: 0.4(95\% CI 0.1,1.31).

Compared to those with a primary level of education, the participants with a secondary and tertiary level of education had 55\%, and $16 \%$ reduced odds of a diagnosis of depression; OR: 0.45 (95\% CI: $0.05,4.23)$, and 0.84 (95\% CI: 0.09, 7.49) respectively.

The odds of being diagnosed with depression among patients with breast cancer among those who were employed had an increased odds of $270 \%$, compared to the unemployed, OR: 3.7(95\% CI: 1.07, 17.27). 
www.yumedtext.com | February-2021 | ISSN: 2582-3264 | https://dx.doi.org/10.46527/2582-3264.133

Compared to the participants who had received hormonal therapy, there was a reduced odds of $48 \%$ of being diagnosed with depression among patients with breast cancer than those not receiving hormonal therapy, OR: $0.52(95 \%$ CI: $0.18,1.42)$.

The findings for using chemotherapy as a mode of treatment were as follows; compared to adjuvant chemotherapy, the participants on neoadjuvant chemotherapy and palliative chemotherapy had $830 \%$ and $850 \%$ increased odds of having a diagnosis of depression among patients with breast cancer. OR: 9.43(95\% CI: 1.5, 185.32) and 9.5(95\% CI: 1.62, 181.81) respectively. This finding was statistically significant.

The odds of being diagnosed with depression among patients with breast cancer among participants who had surgical treatment had a reduced odds of $37 \%$ compared to those who did not have surgical treatment, OR: $0.63(95 \%$ CI: $0.25,1.55)$.

Compared to the early stage of cancer, the participants with late stage of cancer had $61 \%$ increased odds of having a diagnosis of depression among patients with breast cancer, OR: 1.61(95\% CI: 0.63, 4.17) shown in TABLE 4.

TABLE 4. Multivariate logistic regression

\begin{tabular}{|c|c|c|}
\hline Variable & Adjusted ORs (95\% CIs) & P-Value \\
\hline Age & $0.95(0.87,1.03)$ & 0.216 \\
\hline Surg_Tx & $1.73(0.33,9.54)$ & 0.516 \\
\hline Hormonal_Therapy & $0.87(0.24,3.22)$ & 0.839 \\
\hline Marital Status: Married & $4.08(0.7,28.94)$ & 0.13 \\
\hline Employment Status: Employed & $4.29(0.84,33.51)$ & 0.107 \\
\hline Education Level & & \\
\hline Primary & Ref & 0.3 \\
\hline Secondary & $0.23(0.01,3.55)$ & 0.656 \\
\hline Tertiary & $0.52(0.02,8.97)$ & \\
\hline Chemotherapy & & 0.024 \\
\hline Chemotherapy.1 & Ref & 0.041 \\
\hline Chemotherapy.2 & $16.55(2,377.23)$ & \\
\hline Chemotherapy.3 & $20.85(1.46,658.69)$ & \\
\hline
\end{tabular}

* Predictors adjusted for Age, surgical treatment, hormonal therapy, marital status, employment status, education level, and chemotherapy.

** Large CIs due to low cell sizes.

\subsection{Findings of adjusted analysis}

Finding shows that there were 329\% increased odds among the employed of having depression among patients with breast cancer compared to the unemployed OR: $4.29(95 \%$ CI: 0.84, 33.51), and a reduced odds of $13 \%$ among those who receive hormonal therapy compared to those who did not, OR: 0.87(95\%CI: 0.24, 3.22). There were $73 \%$ increased odds of having depression among those who had surgical treatments, compared to those who did not have surgical treatment OR: 1.73(95\% CI: $0.33,9.54$ ) compared to chemotherapy 1 (adjuvant), the participants with chemotherapy 2(non-adjuvant) and chemotherapy 3 (palliative) had 1550\% and 1950\% increased odds having a diagnosis of depression among patients with breast cancer OR:16.55(95\%CI:2,377.23) and 20.85(95\%CI:1.46,658.69) respectively. This finding was statistically significant. Compared 
www.yumedtext.com | February-2021 | ISSN: 2582-3264 | https://dx.doi.org/10.46527/2582-3264.133

to those with a primary level of education, the participants with a secondary and tertiary level of education had $77 \%$ and $48 \%$ reduced odds of a diagnosis of depression, OR: $0.23(95 \%$ CI: $0.01,3.55)$ and $0.52(95 \% \mathrm{CI}: 0.02,8.97)$ respectively.

\section{Discussion}

\subsection{Association of socio-demographic factors and depression among breast cancer patients}

In this study age, education levels, substance abuse, and marital status were found not to be associated with depression among patients with breast cancer. From our study, we found an association between depression and employment with a p-value (0.047). In our study, the mean age was 40 years. Studies by Hassan et al., [11] showed the commonest age of breast cancer is between 40-49 mean ages of 50 years. A recent study in Germany found older patients were 1.2 likely to experience depression than the younger patients. Growing older is one of the major risk factors for breast cancer. In a study in north India mean age of cases and controls was 54.0 years with S.D. \pm 9.7 and 53.8 years with S.D. \pm 9.3 years respectively and the range was 30 80 years. It is also comparable to many of the previous studies. In a study by Afsaneh Bakhtiari et al. [12] the mean age of those with breast cancer were 47.63 years and 49.18 years, and 51.3 years and 48.9 years. Eva Singletaryet al. [13] concluded that if all women less than 65 years of age compared with women aged 65 or older, the relative risk of breast cancer associated with increased age is 5.8 .

In our study the majority of the participants had a tertiary level of education. Low levels of education were found to be a predictor of psychological co morbidity in patients with breast cancer. This somehow might be explained by the fact that patients with higher educational levels have a greater opportunity to be aware of their disease and its related aspects [14]. In a study in South Africa, Kagee et al., [15] reported that the less education the higher the risk of depression among breast cancer patients. A similar finding was reported by Osborne et al., [16]. Our findings reinforcing findings from the existing literature and supporting the assumption that education can be a protective factor in the occurrence of depression and anxiety among women dealing with breast cancer [3]. This finding can be attributed to the fact that women with higher educational status can have better access to information regarding their health condition and be fully aware and understand the treatment plan and what is to be expected from it.

Marital status was one of the associated factors with depression. In our study, there was no statistical significance. Similar findings were reported in studies done by Hassan et al., [11]. A study in North India reported that single women showed a higher prevalence of depression compared to married. A study done in Lagos Nigeria reported that being married is a protective factor and is less likely to develop depression Akechi et al., [17]. Studies have shown that the advantages of employment are weaker in married women, more so if they have children and in preschool. A study done in Malaysia reported that women who were single and unmarried compared to married had better global health status. Chui et al., [18] \& Edib et al., [19].

\subsection{Other factors associated with depression among patients with breast cancer}

The problems experienced by survivors include physical symptoms, psychological reactions, and existential concerns, which potentially disrupt their well-being. The wide range of rates may be due to several factors, including the use of different instruments to assess depression with different psychometric properties, the use of different criteria for defining depression, and differences between included cancer populations concerning cancer type, stage, and treatment modality. Having breast cancer or receiving treatment has been seen as a traumatic experience to people due to its impacts on their self-image and sexual 
www.yumedtext.com | February-2021 | ISSN: 2582-3264 | https://dx.doi.org/10.46527/2582-3264.133

relationship, so most of the breast cancer patients have psychological reactions such as denial, anger, or intense fear toward their disease and treatment process, and many of have psychiatric morbidities. Many of the breast cancer patients experience fatigue, depression, and/or anxiety months to years after their breast cancer diagnosis with these symptoms being associated with greater disability and a poorer quality of life [20]. Adjuvant chemotherapy may lead to an increased risk of depression, anxiety, or both during but not after treatment [21].

The study we carried out at MTRH, found that chemotherapy, employment, and cancer stage had an association with the development of depression among patients with breast cancer. Employment had a p-value of 0.047. Being employed was associated because of various factors: trying to adjust their financial uses in anticipation of treatment, care for their families and periods of being unwell and being out of work and fear of losing their jobs contributed to the distress in this population. A study done in Malaysia reported that the employed had better health globally. Edib et al., [19], Chui et al., [18], found that the unemployed or with low income had better health globally. This was because of welfare assistance from the government, while those with employment and high income are not eligible for Routine screening for depression among breast cancer patients could help enhance early diagnosis of depression and hence management of the disorder. This will in turn help reduce mortality and improve the quality of life of these patients. Studies by Hassan et al., [11] found that MDD is more prevalent among the ethnic minority and low income. Those who were unemployment were unlikely to receive psychiatric treatment and supportive counseling. They felt burdened by cancer treatment and expenses. Being employed has beneficial effects on psychological health. It brings interest, income, fulfillment, social contacts, and status, and provides structure and a sense of control.

Chemotherapy as a mode of treatment for cancer was also found to have an association with the development of depression among patients with breast cancer. Chemotherapy has unpleasant side-effects, such as alopecia and nausea. After long periods of treatment, these side effects are significant and lead to psychological distress. The kinds of chemotherapy depending on the cancer stages are adjuvant, non-adjuvant, and palliative chemotherapy.

Our results show that the use of chemotherapy as a mode of treatment was associated with the development of depression among patients with breast cancer. This was an expected finding and is similar to findings by other authors examining this association among patients with breast cancer. Vahdaninia et al. [22] found that the use of chemotherapy had adverse side effects and psychosocial effects on patients with breast cancer $(\mathrm{p}=0.003)$. A similar study done in Asia found an association between chemotherapy and depression among patients with breast cancer [23]. A similar finding was reported in a study in Iran [24].

Cancer stage was found to have an association with the development of depression among patients with breast cancer ( $\mathrm{p}=0.002$ ). This was an expected finding. Patients who were diagnosed in stage IV of breast cancer were at high risk for depression and anxiety compared to stage I patients. This finding is per a recent study among breast cancer in Greece in which stage IV breast cancer patients were 1.9 ( $\mathrm{p}=0.003)$ more likely to experience depressive symptoms. These findings are similar to a study by Mustian et al. [25]. A similar result was reported in Iran by Novin et al., [24]. A study done in China found that there was no association between the breast cancer stage and the development of depression among patients with breast cancer $(\mathrm{p}=0.192)$ [26]. 
www.yumedtext.com | February-2021 | ISSN: 2582-3264 | https://dx.doi.org/10.46527/2582-3264.133

Surgical treatment as a mode of treatment was found not to have an association with the development of depression among patients with breast cancer, this was the findings from our study, the results were unexpected finding $\mathrm{P}=0.315$. Mastectomy can cause scars and disfigurement, which can lead to a perception of becoming less attractive, poor body image which is associated with depression. Undergoing active cancer treatment, specific drug treatments Beatty and Kissane, [27], worries regarding fear of death and disease recurrence, altered body image [22], alteration of sexuality and attractiveness. A study done in Karachi hospital found that going through mastectomy leads to moderate to severe depression among patients with breast cancer, this was primarily because one feels incomplete and insecure after losing a part of themselves Sara Khan et al., [28].

Hormonal therapy is one of the modes of treatment used to treat patients with breast cancer. Tamoxifen is mainly used in our setting. Tamoxifen is an estrogen hormone adjuvant used for more than 25 years to treat women with breast cancer with proven efficiency for the reduction of mortality and in preventing recurrences. The use of tamoxifen, compared to no treatment, reduces the risk of recurrence for about 15 years [29]. The factors usually indicated as those contributing to non-adherence in breast cancer patients include the therapy's adverse effects. Hormone therapy, including tamoxifen, may cause hot flashes, fluid retention, bleeding, skin rashes, vaginal itching, and dryness, the risk of endometrial cancer, joint pain, or deep vein thrombosis, among other (Early Breast Cancer Trialists' Collaborative Group (EBCTCG) [30] findings from our study was that there was no association with the development of depression among patients with breast cancer. This finding was unexpected. Several studies carried out in parts of the world found there was an association. Findings from a study done in China found out that hormone receptor-positive tumors are generally associated with a better prognosis compared to the hormone negative receptors and that the incidence of depression was low.

During the study period, we found the participant at the first encounter (admission) that had varied forms of depression, ranging from mild to severe forms of depression. These participants were immediately referred to a psychiatrist, some of the patients were started on antidepressants, while the majority were referred to a psychologist and were started on psychotherapies. We followed up on these participants after six months and twelve months. We noticed marked improvements in their depression profile.

\section{Conclusion}

This study sought to find the factors associated with MTRH to facilitate the diagnosis of depression among this population. Findings from our study showed that being employed, the use of chemotherapy and the cancer stage were significantly associated with having depression.

\section{Recommendations}

The above information obtained from this study has major clinical significance, mainly in the development of guidelines for early identification of depression among breast cancer patients at MTRH.

- Screening for depression should be initiated for a patient with cancer at all point $\mathrm{s}$ of interaction with patients.

- Early psychological support should be incorporated into the management of these patients

- More studies with a larger population to determine the associated factors with other psychiatric morbidities.

- More studies on the association between and chemotherapy as a mode of treatment should be done. 


\section{REFERENCES}

1. Massie MJ. Prevalence of depression in patients with cancer. J Natl Cancer InstMonogr. 2004;2004(32):57-71.

2. Ballantyne JC, Fishman SM, Rathmell JP, et al. 4th edn. Management of Pain. Philadelphia, Pa: Lippincott Williams \& Wilkins, USA; 2009.

3. Hopwood P, Sumo G, Mills J, et al. The course of anxiety and depression over 5 years of follow-up and risk factors in women with early breast cancer: results from the UK Standardization of Radiotherapy Trials (Start). Breast. 2010;19(2):84-91.

4. Noal S, Levy C, Hardouin A. One-year longitudinal study of fatigue, cognitive functions, and quality of life after adjuvant radiotherapy for breast cancer. Int J Radiat Oncol Biol Phys. 2011;81(3):795-803.

5. Tenge CN, Kuremu RT, Buziba NG, et al. Burden and Pattern of Cancer in Western Kenya. East Afr Med J. 2009;86(1):7-10.

6. Pyter LM, Cochrane SF, Ouwenga RL, et al. Mammary tumors induce select cognitive impairments. Brain Behav Immun. 2010;24(6):903-7.

7. Lewis FM, Fletcher KA, Cochrane BB, et al. Predictors of Depressed Mood in Spouses of Women with Breast Cancer. J Clin Oncol. 2008;26(8):1289-95.

8. Jemal A, Center M, DeSantis C, et al. Global patterns of cancer incidence and mortality rates and trends. Cancer Epidemiol Biomarkers Prev. 2010;19(8):1893-907.

9. Bagby RM, Ryder AG, Schuller DR, et al. The Hamilton Depression Rating Scale: Has the gold standard become a lead weight. Am J Psychiatry. 2004;161(12):2163-77.

10. Edwards BC, Lambert MJ, Moran PW, et al. A meta-analytic comparison of the Beck Depression Inventory and the Hamilton Rating Scale for Depression as measures of treatment outcome. Br J Clin Psycho. 1984;23(Pt 2):93-9.

11. Hassan MR, Shah SA, Ghazi HF, et al. Anxiety, and Depression among Breast Cancer in an Urban setting in Malaysia. Asian Pac J Cancer Prev. 2015;16:4031-5.

12. Nikpour M, Hajian-Tilaki K, Bakhtiari A. Risk Assessment for Breast Cancer Development and Its Clinical Impact on Screening Performance in Iranian Women. Cancer Manag Res. 2019;11:10073-82.

13. Singletary SE. Rating the risk factors for breast cancer. Ann Surg. 2003;237(4):474-82.

14. Mehnert A, Koch U. Psychological comorbidity and health-related quality of life and its association with awareness, utilization, and need for psychosocial support in a cancer register-based sample of long-term breast cancer survivors. J Psychosom Res. 2008;64:383-91.

15. Magidson JF, Saal W, Nel A, et al. Relationship between depressive symptoms, alcohol use, and antiretroviral therapy adherence among HIV-infected, clinic-attending patients in South Africa. J Health Psychol. 2017;22(11):1426-33.

16. Osborne R, Elsworth G, Hopper J. Age-specific norms and determinants of anxiety and depression in 731 women with breast cancer recruited through a population-based cancer registry. Eur J Cancer. 2003;39:755-62.

17. Akechi T, Kugaya A, Okamuna H, et al. Correlates of depressed mood in ambulatory head and neck patients. Psychooncology. 1999;8(6):494-9.

18. Chui PL, Abdullah KL, Wong LP. Quality of Life in CAM and Non-CAM Users among Breast Cancer Patients during Chemotherapy in Malaysia. PLoS One 2015;10.

19. Edib Z, Kumarasamy V, BintiAbdullah N. () Most prevalent unmet supportive care needs and quality of life of breast cancer patients in a tertiary hospital in Malaysia. Health Qual Life Outcomes. 2016;14:26. 
20. Rogers LQ, Hopkins-Price P, Vicari S, et al. A randomized trial to increase physical activity in breast cancer survivors. Med Sci Sports Exerc. 2009;41(4):935-46.

21. Burgess C, Cornelius V, Love S, et al. () Depression and anxiety in women with early breast cancer: a five-year observational cohort study. BMJ. 2005;330:402-705.

22. Vahdaninia M, Omidvari S, Montazeri A. What do predict anxiety and depression in breast cancer patients? A followup study. Soc Psychiatry Psychiatr Epidemiol. 2010;45(3):355-61.

23. Eversley R, Etrin D, Dibble S. Post-treatment among ethnic minority breast cancer survivors. Oncol Nurs Forum. 2005;32(2):250-6.

24. Nikbakhsh N, Moudi S, Abbasian S, et al. Prevalence of depression and anxiety among cancer patients. Caspian J Intern Med. 2014;5(3):167-70.

25. Mustian KM, Sprod LK, Janelsins M, et al. Exercise Recommendations for Cancer-Related Fatigue, Cognitive Impairment, Sleep problems, Depression, Pain, Anxiety, and Physical Dysfunction: A Review. Oncol Hematol Rev. 2012;8(2):81-8.

26. Hong JS, Tian J. Prevalence of anxiety and depression and their risk factors in Chinese cancer patients. Supportive care cancer. 2014;22(2):453-9.

27. Beatty L \& Kissane D. Anxiety and depression in women with breast cancer. Cancer Forum. 2017;41(1):55-61.

28. Khan S, Khan NA, Rehman AU, et al. Levels of Depression and Anxiety Post-Mastectomy in Breast Cancer Patients at a Public Sector Hospital in Karachi. Asian Pac J Cancer Prev. 2016;17(3):1337-40.

29. Ma AM, Barone J, Wallis AE, et al. () Noncompliance with adjuvant radiation, chemotherapy, or hormonal therapy in breast cancer patients. Am J Surg. 2008;196(4):500-4.

30. Early Breast Cancer Trialists' Collaborative Group (EBCTCG). Effects of chemotherapy and hormonal therapy for early breast cancer on recurrence and 15-year survival: an overview of the randomized trials. Lancet. 2005;365:1687717. 